\title{
Method to Calculate Dynamic Accessibility of Urban Bus Rapid Transit System
}

\author{
Rongge GUO ${ }^{1, a^{*}}$, Jihua $\mathrm{HU}^{2, \mathrm{~b}}$, Wei GUAN ${ }^{1, \mathrm{c}}$, Jie XIONG ${ }^{1, \mathrm{~d}}$ \\ ${ }^{1}$ MOE Key Laboratory for Urban Transportation Complex Systems Theory and Technology, \\ Beijing Jiaotong University, Beijing, 100044, China \\ ${ }^{2}$ College of Engineering, Sun Yet-Sen University, Guangzhou, 510006, China

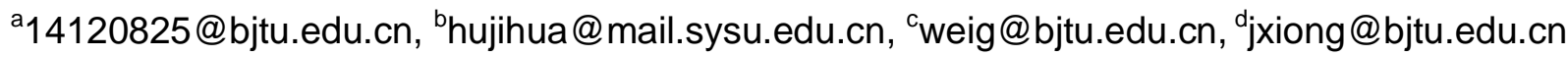

Keywords: transit; dynamic accessibility; enhanced two-step floating catchment technique; bus rapid transit; distance-decay function

Abstract. Bus rapid transit (BRT) is an important transit mode in the urban transportation system, especially in improving the urban transit's operating efficiency. The accessibility of BRT is an important factor to measure its service level, however, most existing methods of calculating the accessibility can hardly reflect its real-time changes due to the lack of consideration for the time-varied vehicle capacity or the passenger's walking distance. Therefore, a new enhanced two-step floating catchment technique (E2SFCA) is proposed to calculate the dynamic accessibility instead of the traditional two-step floating catchment technique (2SFCA) by adding a distance-decay function. Two districts in Guangzhou, i.e. TianHe and HuangPu are presented as real-life examples to validate the effectiveness of E2SFCA. Based on the results, some conclusions and suggestions are provided to improve the service of BRT. The related results also confirm that E2SFCA has a significant advantage to the planning and operation of BRT.

\section{Introduction}

Traffic jams are very common in big cities nowadays, as the fast growth of urban population and private cars. To alleviate the traffic pressure, the bus rapid transit (BRT) system has been developed in many big cities. Various calculation methods of transit accessibility have been proposed (Chen et al.2008); Chen (2015) used the GIS spatial analysis the transit accessibility. Luo (2003) put forward two step floating catchment technique(2SFCA)to research the accessibility of medical care space. By modifying the 2SFCA, Langford (2012) measured the transit accessibility of Merthyr.

Compared with the design of ordinary bus lines and stops, the design of BRT focuses on the network integrity and real-time vehicle information. Thus, the dynamic accessibility measure is more suitable for BRT. The walking distance and transit coverage are two important factors for the dynamic accessibility calculation of BRT. O'Sullivan (1996) found that the transit demand was diminished by $70 \%$ with the walking distance increasing from 200 to $400 \mathrm{~m}$. Zhao (2003) observed that the optimal walking distance should not more than 550m. Hess (2012) suggested that the optimal walking distance was $400 \mathrm{~m}$.

To our best knowledge, few existing researches consider the dynamic accessibility. Moreover, 2SFCA method ignores the distance attenuation factor. Therefore, the results may not reflect the actual case. On the basic of the traditional 2SFCA method, this paper proposed an enhanced two-step floating catchment technique (E2SFCA) to calculate the accessibility of BRT by considering a distance-decay function.

\section{BRT Dynamic Accessibility}

Accessibility is usually defined as "the degree of convenience that people can reach some certain services from their origination" ( $\mathrm{Lu}$ et al.2009). For public transportation system, an accurate accessibility measurement is an important factor in evaluating the service level of transit, and it is also provide guidance to the construction and improvement of the transit. 
This paper defines the concept of the transit dynamic accessibility as "a real-time measurement of the difficulty that people can reach the transit service in a relatively stable transit operation environment", and it is affected by the number of buses in service and the transit demand in real time. Comparing with static accessibility, the dynamic calculating method highlights the instantaneity and flexibility of the measurement. Moreover, the indicator of dynamic accessibility is also accessibility influenced by some other factors, such as geographical location, passenger flow, vehicle capacity and traffic density.

\section{Modification of E2SFCA Based on BRT}

Introduction about 2SFCA. 2SFCA (Luo et al.2003) is a static binary method. It is based on the assumption that all the service points within a scope have the same attraction.

Luo (2009) proposed an enhanced 2SFCA (E2SFCA) method by adding a distance-decay factor. This modification indicates that the transit accessibility changes versus service distance. E2SFCA is a dynamic method, which improves the effect of walking distance on the transit demand, and it reflects the real case better.

Calculation of the Attenuation Coefficient. It is obvious that the passengers also less to be attracted by distant stops in the transit service area. Thus, E2SFCA has improved 2SFCA by considering a distance-decay weighting coefficient $W_{k j}$. By this, the ratio of transit supply and demand $R_{j}$ and accessibility metric $A_{k}$, are formulated as Eq. (1)-(2), respectively:

$$
\begin{aligned}
& R_{j}=\frac{S_{j}}{\sum_{k \in\left(d_{k j} \leq d_{\max }\right)} P_{k} \times W_{k j}} \\
& A_{k}=\sum_{j \in\left(d_{k j} \leq d_{\max }\right)} R_{j} \times W_{k j}
\end{aligned}
$$

where:

$S_{j}:$ service quantity provided by stop $j$

$P_{k}:$ transit demand assembled at point $k$ in the service area of stop $j$

$d_{k j}:$ distance from point $k$ to stop $j$

Three formulations of the distance-decay weighting coefficient $W_{k j}$, are given as follows:

Linear decay

$$
\begin{aligned}
& W_{k j}=\frac{\left(d_{\text {max }}-d_{k j}\right)}{d_{\text {max }}\left(d_{k j} \leq d_{\text {max }}\right)} \\
& W_{k j}=0\left(d_{k j}>d_{\text {max }}\right)
\end{aligned}
$$

Gauss decay

$W_{k j}=\operatorname{Exp}\left(-d_{k j}^{2} / d_{\text {pass }}^{2}\right)$

$d_{\text {pass }}=200$

Butterworth filter 


$$
\begin{gathered}
W_{k j}=\sqrt{\frac{1}{1+\varepsilon\left(d_{k j} / d_{\text {pass }}\right)^{n}}} \\
\left(\varepsilon=1, d_{\text {pass }}=250, n=6\right)
\end{gathered}
$$

According to Zhao's (2003) study, the bus occupancy decreases sharply when walking distance exceeds 91 meters, and it is nearly to be zero when the walking distance is above 580 meters. The change of the bus occupancy versus walking distance is similar to the Butterworth curve, thus Butterworth filter is used to calculate distance decay factor.

Modification of E2SFCA Based on the Characteristics of BRT. As shown in Fig.1, E2SFCA needs to consider the relationships between bus stop and public transit service.

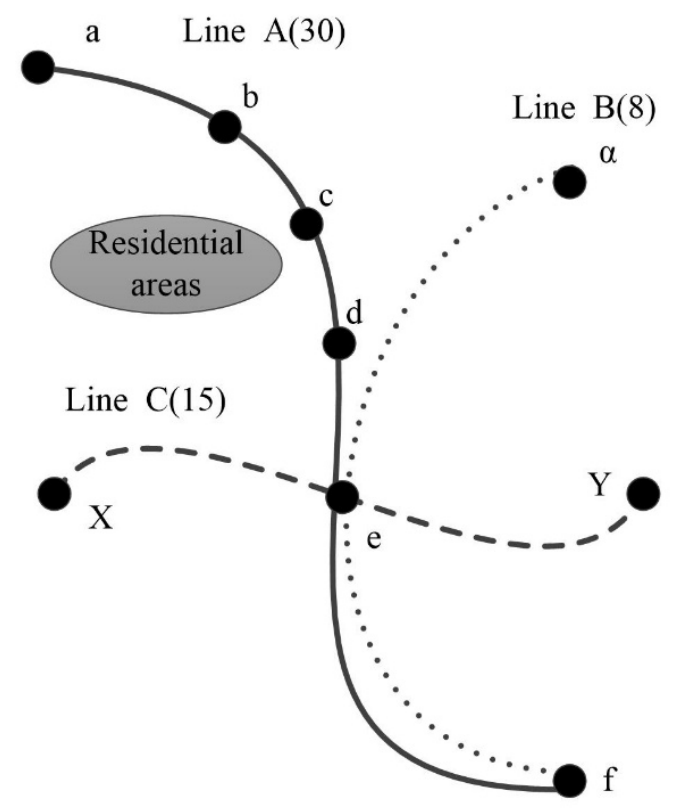

Bus line (daily departure frequency) Bus station

Fig. 1 The BRT lines

Fig. 1 describes a typical situation, where there are three bus routes, nine stops and a single population centroid (transit demand). All the bus stops are within the service distance range. Since there exist both boarding and alighting demands at each stop, the transit supply provided by buses at different stops are different. If this factor is set to be a constant, the transit accessibility will be misestimated.

Moreover, the stops near to passengers are likely to be chosen as boarding stops. It is worth noticing that when calculating the accessibility metric $A_{k}$, for each demand point $k$, the distance-decay weighting $W_{k j}$, needs to be multiplied by each ratio of transit supply and demand $R_{j}$ for each stop $j$. By this, the main steps of the modified E2SFCA based on the characteristic of BRT are given as follows.

Step1: Calculating the transit supply at stop $j$ by recording the number of boarding and alighting passengers, and the number of buses of vehicle type $i$, which are represented respectively by $S_{j}^{u p}, S_{j}^{\text {down }}$, and $N_{i}$. The maximum vehicle capacity of type $i$ is represented by $p_{i}$. The calculation of $S_{j}$ and $R_{j}$ are given as follows:

$$
S_{j}^{s}=\sum_{i \in I}\left(N_{i} \times p_{i}-\sum_{m=1}^{j-1}\left(S_{m}^{u p}-S_{m}^{d o w n}\right)\right)
$$




$$
R_{j}^{s}=\frac{S_{j}^{s}}{\sum_{k \in\left(d_{k j} \leq d_{\max }\right)} P_{k} \times W_{k j}}\left(s \in S_{j}\right)
$$

Step2: Calculating the accessibility of demand $k$ based on $d_{k j(s)}^{\min }$ (the shortest distance from the transit demand point $k$ and the occupied stop $j$ ):

$$
A_{k}=\sum_{s} R_{j}^{s} \times w_{k j}\left(\forall s: d_{k j(s)}^{\min }\right)
$$

\section{A Case Study: Transit System Accessibility TianHe and HuangPu Districts in Guangzhou}

Introduction. A real-life example based on TianHe district and HuangPu district in Guangzhou is presented to analyze the BRT stop accessibility and the surrounding communities through two districts. The BRT stops as shown in Fig.2.

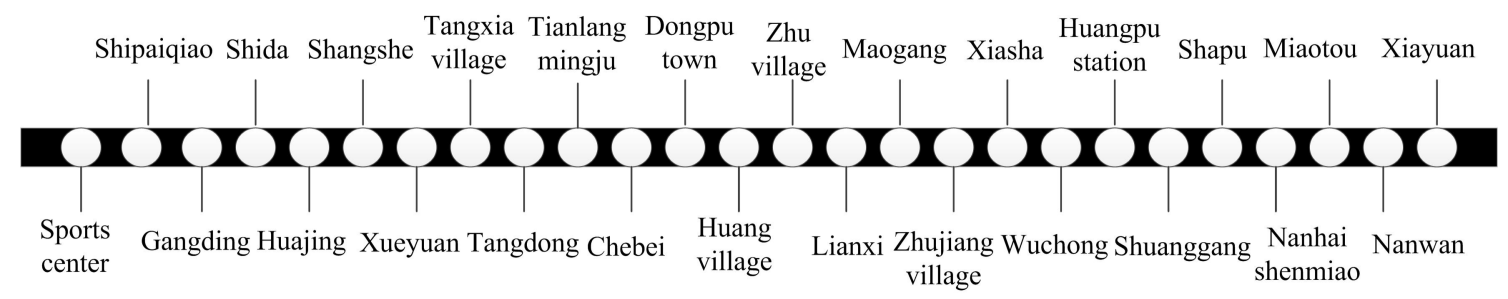

Fig.2 BRT stops

\section{Accessibility Calculation}

(1) Basic data

The information of the BRT stop mainly includes: latitude and longitude, recording time, the number of passengers boarding and alighting, and the number of vehicles. The information of the community mainly includes the geographic location, the information of BRT stops within the community's distance scope, the walking distance to the BRT stop and the attenuation coefficient. The information of each type of the bus is given in Table 1 .

Table 1 Information of each type of the bus

\begin{tabular}{cccc}
\hline bus type & seating & standing area(square meter) & carrying capacity(person) \\
\hline 29seats & 29 & 15.3 & 75 \\
33seats & 33 & 14 & 75 \\
\hline
\end{tabular}

(2)Accessibility results

Fig. 3 is the flow chart of calculating the dynamic accessibility of the BRT station and the community: 


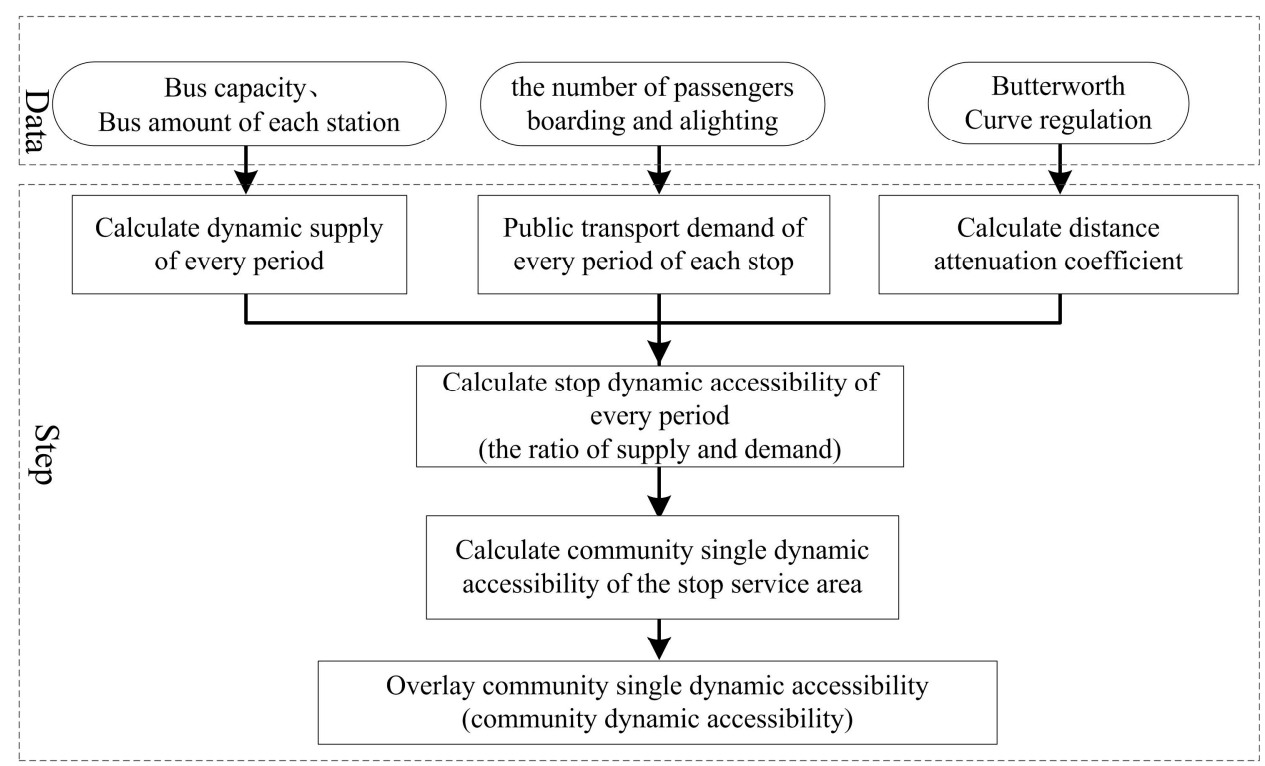

Fig.3 Dynamic accessibility calculation steps of BRT stops and districts

According to the dynamic accessibility calculation steps in Fig.3, the results during the period of 8:30-9:00 a.m. on a weekday are showed in Table 2 and Table 3.

Table 2 BRT stops dynamic accessibility

\begin{tabular}{|c|c|c|c|c|}
\hline stop & time & $\begin{array}{l}\text { card } \\
\text { amount }\end{array}$ & passenger capacity & accessibility \\
\hline Xiayuan & $\begin{array}{c}8: 30-9: 0 \\
0\end{array}$ & 124 & 2175 & 17.54032258 \\
\hline Nanwan & $\begin{array}{c}8: 30-9: 0 \\
0\end{array}$ & 13 & 2054 & 157.9615385 \\
\hline Miaotou & $\begin{array}{c}8: 30-9: 0 \\
0\end{array}$ & 25 & 1975 & 78.98758621 \\
\hline $\begin{array}{c}\text { Nanhaishenm } \\
\text { iao }\end{array}$ & $\begin{array}{c}8: 30-9: 0 \\
0\end{array}$ & 69 & 1743 & 25.2625473 \\
\hline Shapu & $\begin{array}{c}8: 30-9: 0 \\
0\end{array}$ & 22 & 1268 & 57.64854456 \\
\hline Shuangang & $\begin{array}{c}8: 30-9: 0 \\
0\end{array}$ & 74 & 1523 & 20.58475359 \\
\hline $\begin{array}{l}\text { Huangpu } \\
\text { station }\end{array}$ & $\begin{array}{c}8: 30-9: 0 \\
0\end{array}$ & 25 & 939 & 37.57752432 \\
\hline Shida & $\begin{array}{c}8: 30-9: 0 \\
0\end{array}$ & 97 & 1795 & 18.50321763 \\
\hline Gangding & $\begin{array}{c}8: 30-9: 0 \\
0\end{array}$ & 487 & 3556 & 7.302211003 \\
\hline Shipaiqiao & $\begin{array}{c}8: 30-9: 0 \\
0\end{array}$ & 9 & 4263 & 73.6787924 \\
\hline Sports center & $\begin{array}{c}8: 30-9: 0 \\
0\end{array}$ & 54 & 5884 & 108.9674306 \\
\hline
\end{tabular}


Table 3 Districts dynamic accessibility

\begin{tabular}{|c|c|c|c|c|c|c|}
\hline $\begin{array}{c}\text { community } \\
\text { name }\end{array}$ & adjacent stop & $\begin{array}{l}\text { walking } \\
\text { distance }\end{array}$ & $\begin{array}{c}\text { distance } \\
\text { decay factor }\end{array}$ & $\begin{array}{c}\text { stop } \\
\text { accessibility }\end{array}$ & $\begin{array}{c}\text { signal } \\
\text { accessibility }\end{array}$ & $\begin{array}{c}\text { accessibilit } \\
\mathrm{y}\end{array}$ \\
\hline Sports west & Sports center & 500 & $\begin{array}{c}0.124034 \\
735\end{array}$ & $\begin{array}{c}51.355569 \\
37\end{array}$ & $\begin{array}{c}6.369874 \\
438\end{array}$ & $\begin{array}{c}6.369874 \\
43\end{array}$ \\
\hline Yunan & Tangdong & 503 & $\begin{array}{c}0.121861 \\
689\end{array}$ & $\begin{array}{c}1.286073 \\
096\end{array}$ & $\begin{array}{c}0.156723 \\
04\end{array}$ & $\begin{array}{c}0.156723 \\
04\end{array}$ \\
\hline Fuli & $\begin{array}{c}\text { Tianlangming } \\
\mathrm{ju}\end{array}$ & 324 & 0.4174508 & $\begin{array}{c}7.122300 \\
342\end{array}$ & $\begin{array}{c}2.973209 \\
976\end{array}$ & $\begin{array}{c}2.973209 \\
97\end{array}$ \\
\hline Xihua garden & $\begin{array}{c}\text { Tianlangming } \\
\text { ju } \\
\text { Chebei }\end{array}$ & 444 & $\begin{array}{c}0.175735 \\
393 \\
0.134872 \\
929\end{array}$ & $\begin{array}{c}7.122300 \\
342 \\
2.209005 \\
006\end{array}$ & $\begin{array}{c}1.251640 \\
25 \\
0.297934 \\
975\end{array}$ & $\begin{array}{c}1.549575 \\
22\end{array}$ \\
\hline Jujing garden & $\begin{array}{l}\text { Tangxiacun } \\
\text { Tangdong }\end{array}$ & 267 & $\begin{array}{c}0.191545 \\
232 \\
0.634491 \\
943\end{array}$ & $\begin{array}{c}3.030470 \\
522 \\
1.286073 \\
096\end{array}$ & $\begin{array}{c}0.580472 \\
179 \\
0.816003 \\
017\end{array}$ & $\begin{array}{c}1.396475 \\
19\end{array}$ \\
\hline $\begin{array}{c}\text { Xiangxin } \\
\text { garden }\end{array}$ & Shuanggang & 309 & $\begin{array}{c}0.468014 \\
794\end{array}$ & $\begin{array}{c}11.652682 \\
99\end{array}$ & $\begin{array}{c}5.453628 \\
03\end{array}$ & $\begin{array}{c}5.453628 \\
03\end{array}$ \\
\hline Shapu village & Shapu & 420 & $\begin{array}{c}0.206358 \\
578\end{array}$ & $\begin{array}{c}34.905040 \\
17\end{array}$ & $\begin{array}{c}7.202954 \\
455\end{array}$ & $\begin{array}{c}7.202954 \\
45\end{array}$ \\
\hline Bochuan & $\begin{array}{c}\text { Nanhaishenm } \\
\text { iao }\end{array}$ & 207 & 0.86965008 & $\begin{array}{c}16.363255 \\
2\end{array}$ & $\begin{array}{c}14.230306 \\
19\end{array}$ & $\begin{array}{c}14.230306 \\
1\end{array}$ \\
\hline
\end{tabular}

(3) The design and analysis of accessibility map

For analyzing the changes of the results, accessibility maps are accomplished by ArcGIS. As shown in Fig.4 and Fig.5, circular classification symbol and histogram are used to represent stop accessibility and community accessibility.

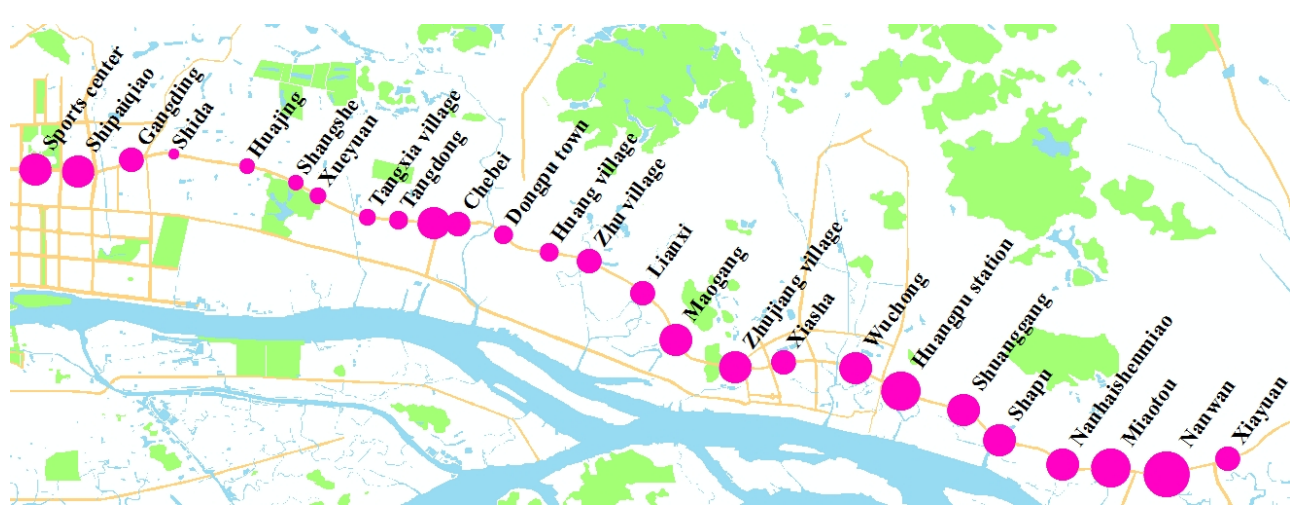

Fig.4 BRT stops dynamic accessibility GIS map

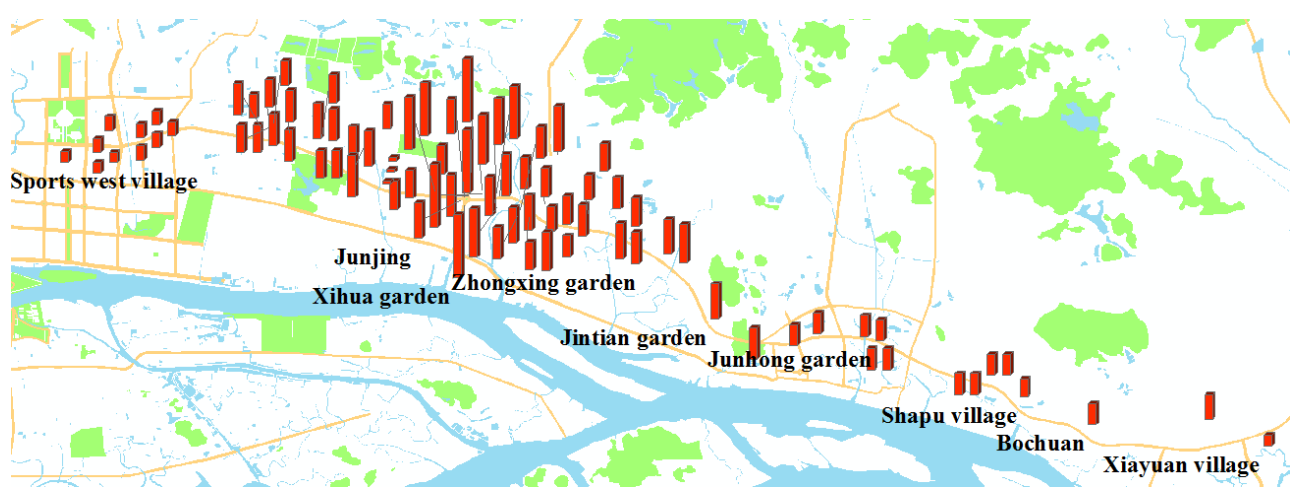

Fig.5 Districts dynamic accessibility GIS map 
The results of the stop accessibility show that the accessibility of station on both ends is low, while the accessibility in the middle part is high. The different results can be attributed to the different stop distribution, geographical location, and population density of the stations. On the right side of the bus line are industrial parks, which belong to sparsely populated region and the population type there is also single; while on the left side of the bus line are commercial districts, where the transit network is complex and the transit service is also improved, thus the BRT dynamic accessibilities there are at a higher level. The middle part of the bus line is a residential area, where the population density is larger. Moreover, this area belongs to suburban areas, where the economic and the relative infrastructure are not well developed. It makes the BRT dynamic accessibility there quite low.

The results of the community accessibility show that the accessibility in the central district is higher than villages at the endpoints. The main reason is that a large number of migrant workers reside in rural-urban continuum. To meet the people's travel demands, BRT stops are densely distributed, thus the community dynamic accessibility is high. While the villages regions at the endpoints are industrial and commercial districts, causing that the population distribution is unbalanced. The villages are distributed in pairs of spots, thus the distance attenuation coefficient is larger and the accessibilities are lower. Based on the above analysis, the example validates that BRT can alleviate the pressure of the residents, and E2SFCA is effectiveness.

In addition, since the accessibilities of stops and communities are reversible, demand and supply are not balance in some areas. The transit demand from the communities in the middle of the bus line cannot be satisfied at peak period, while there exists oversupply on the endpoints of the line.

Optimization and Analysis. Based on the assumption that there are enough vehicles, the dynamic accessibility information is used to guide the operation of BRT by adjusting the departure time and the headway in real-time.

In reality, the BRT service includes two types, BRT short-line service and BRT express services. It is more reasonable to combine these two types together to do the optimization rather than handling them separately. For example, the optimal solutions of Line B1 and some related suggestions are as follows:

(1)Increasing the BRT express service and BRT short-line service

Considering the dynamic accessibility of each BRT stop, the operations of increasing the BRT express service and BRT short-line service to serve the low accessibility stops and adjusting the change with the change of demands.

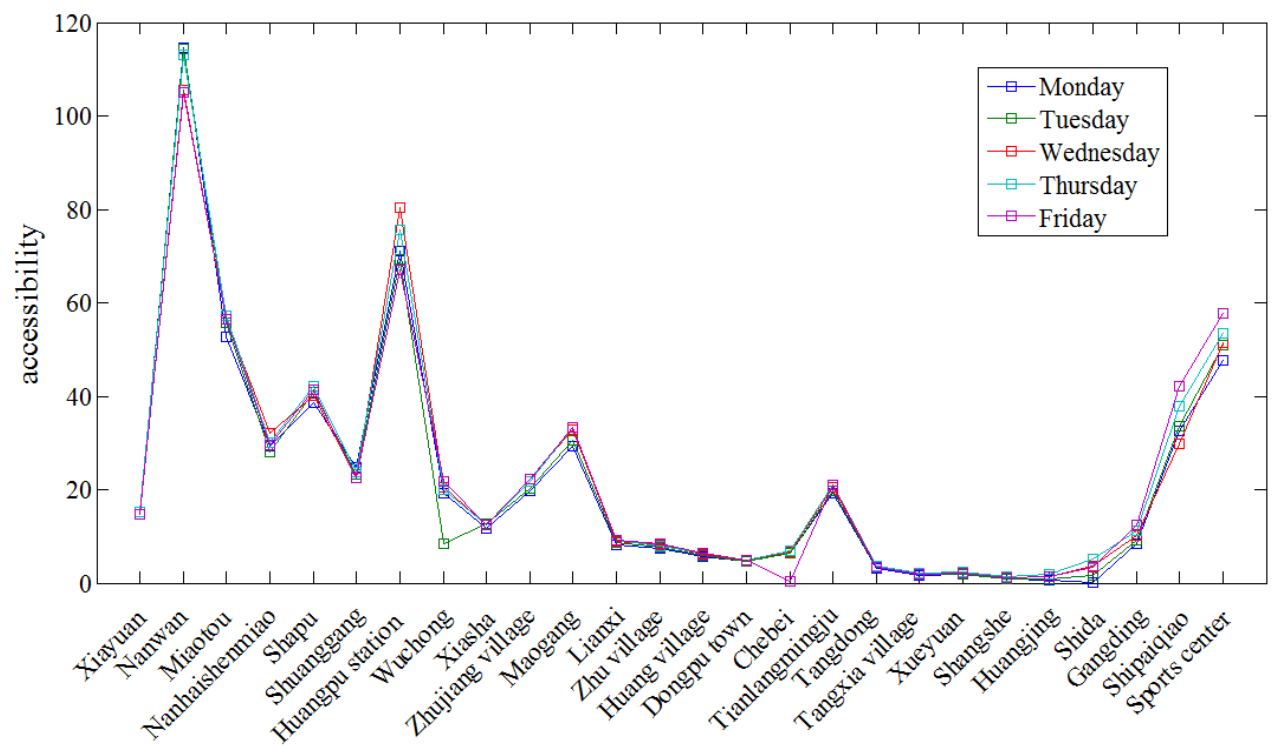

Fig.6 B1 stop dynamic accessibility during morning rush

From Fig.6, B1 express is designed according to the transit supply and demand at each stop, and is formulated as : Xia Yuan - Shuanggang - Xiasha - LianXi - Dongpu town - Chebei - 
Tangdong-Tangxia village - Xueyuan - Shangshe - Huajing - Gangding - Sports center; the operating hours are 7:00-9:00.

(2)Adjustments of BRT express stops and BRT short-line service stops

The vehicle schedule, line length and stop spacing for existing express and short routes should be adjusted considering the dynamic accessibility.

(1) B1 express service

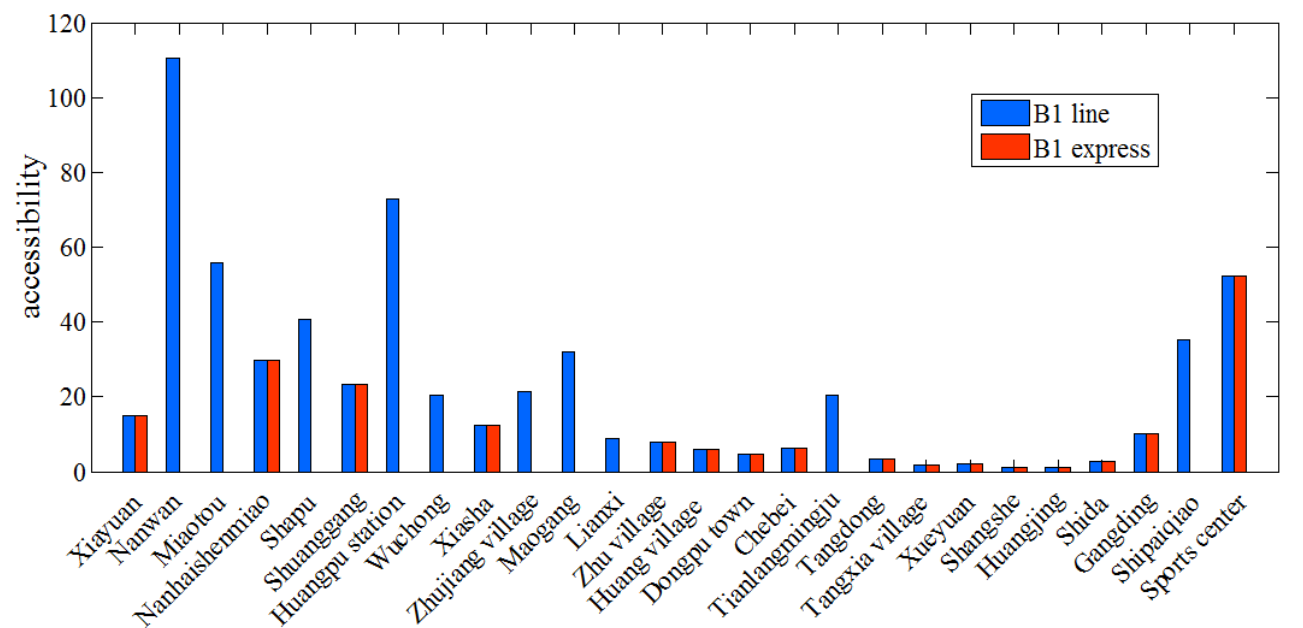

Fig.7 Comparison of accessibility between B1 line and B1 express during weekly morning rush

As shown in Fig.7, comparing with the stop accessibilities of morning peak, B1 express stops should be adjusted by removing stops with higher accessibility, such as Nanhaishenmiao , Shuanggang、Sports center, and adding lower accessibility stop like Lianxi. Therefore, the new B1 express is: Xiayuan - Xiasha - LianXi - Zhu village - Huang village -Dongpu town - ChebeiTangdong - Tangxia village- Xueyuan - Shangshe - Huajing- Shida-Gangding.

(2) B1 short-line service

B1 short route passes the residential area of TianHe district, and the line chart is shown in Fig.8:

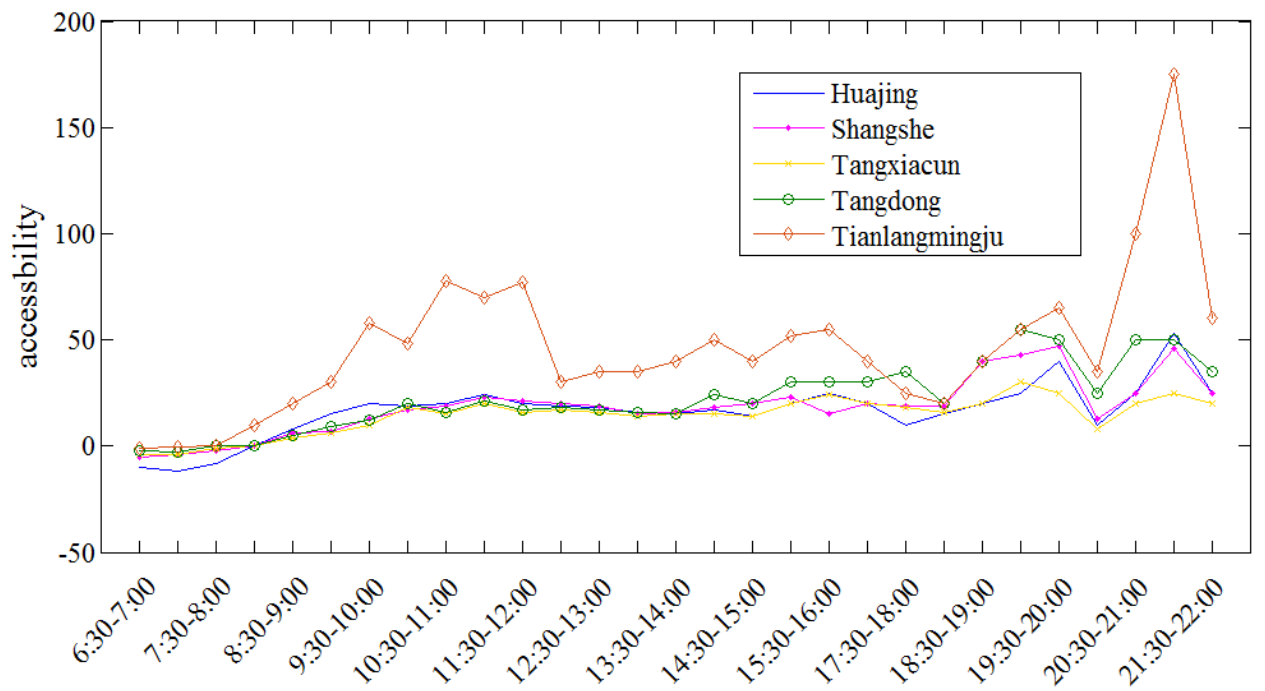

Fig. 8 B1 stop dynamic accessibility all the time

Taking the Tianlangmingju stop as an example, the accessibility of this stop is lower in morning peak, evening peak and during20:00 - 22:00. Thus, B1 short route only needs to stop at this stop during these three periods.

(3)Reducing the headway and adjusting the departure time 
The operation of BRT express and BRT short trip are based on the real-time vehicle capacity. In order to allocate resources reasonably, adjusting headway and departure time based on the stop dynamic accessibility. For instance, as shown in Fig.9, the operating time for B1 express is from 7:00 to $22: 00$.

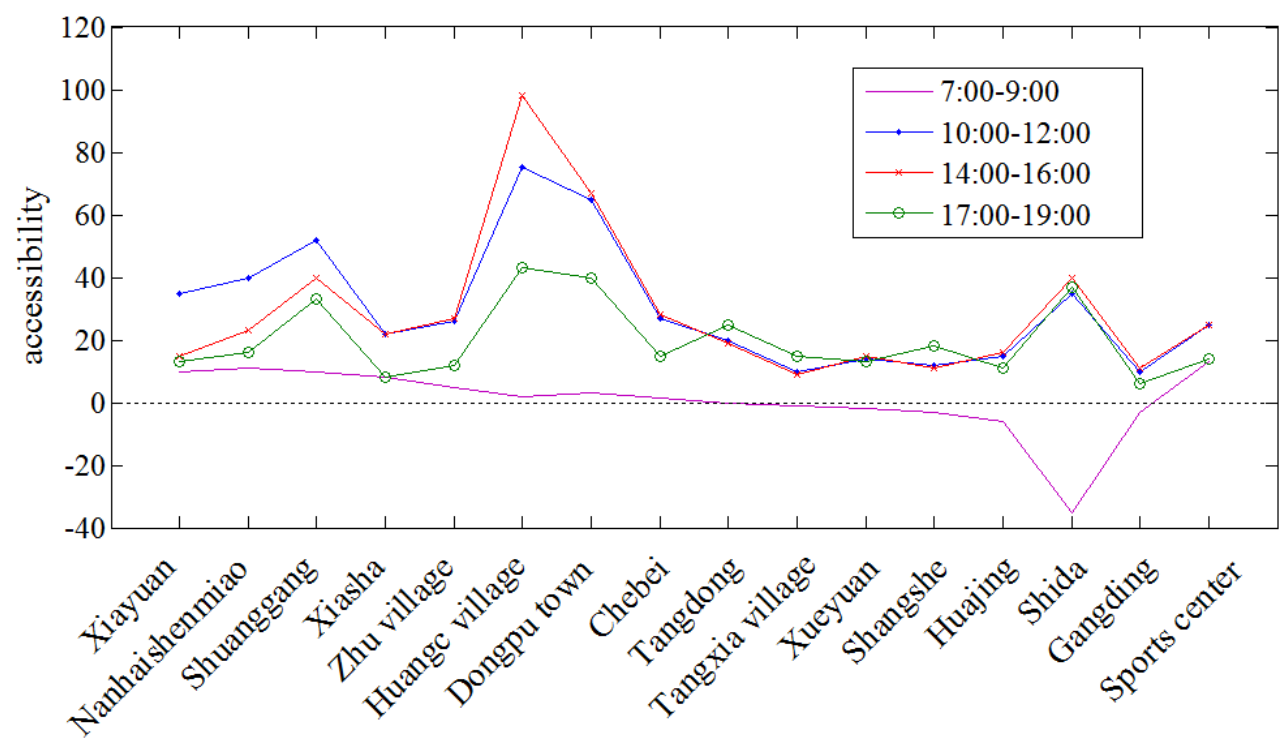

Fig.9 Dynamic accessibility of B1 fast stops all the time

Because the stops accessibilities are lower during peak time, the headway during these periods should be shortened. In addition, since a stop's accessibility is higher during 10:00-12:00 and 14:00-16:00, it is suitable to increase the headway during these periods.

\section{Conclusions}

This paper puts forward an E2SFCA method to calculate the dynamic accessibility of BRT by considering the impact of the walking distance on the transit demand. Two districts in Guangzhou, e.t. TianHe and HuangPu are presented as real-life examples to validate the feasibility of the method. The related results are calculated, and then some visualized maps based on GIS follow. In a certain range of allowable error, the results show that E2SFCA can calculate the BRT dynamic accessibility accurately and reflects the real case better. The results are of great significance to optimization and construction of BRT system.

\section{Acknowledgement}

National Natural Science Foundation Project(71131001)

\section{References}

[1] J. Chen, F. Lu, C. X. Cheng, Advance in Accessibility Evaluation Approaches and Applications [J]. Progress in Geography, 2008, 26(5):100-110.

[2] Y. Y. Chen, P. Y. Wei, J. H. Lai. A calculation method of area public transit accessibility based on GIS [J].Journal of transportation systems engineering and information technology,2015, 15(2):61:67.

[3] Luo W, Wang F. Measures of spatial accessibility to health care in a GIS environment: synthesis and a case study in the Chicago region[J]. Environment and Planning B Planning and Design, 2003 (30): 865-884. 
[4] Langford M, Fry R, Higgs G. Measuring transit system accessibility using a modified two-step floating catchment technique[J].International Journal of Geographical Information Science, 2012, 26(2):193-214.

[5] O'Sullivan S, Morrall J. Walking distances to and from light-rail transit stations [J]. Transportation research record: journal of the transportation research board, 1996, 1538(1):19-26.

[6] Zhao F, Chow L F, Li M T. Forecasting transit walk accessibility: regression model alternative to buffer method[J].Transportation Research Record: Journal of the Transportation Research Board, 2003, 1835(1): 34-41.

[7] Hess D B. Walking to the bus: perceived versus actual walking distance to bus stops for older adults[J]. Transportation, 2012, 39(2):247-266.

[8] H. P. Lu, J. F. Wang, Y. B. Zhang. City traffic accessibility model and its application in traffic planning [J]. Tsinghua Univ(Sci \& Tech) ,2009(6):781-785.

[9] W L, Y Q. An enhanced two-step floating catchment area (E2SFCA) method for measuring spatial accessibility to primary care physicians[J]. Health \& Place, 2009, 15(4):1100-1107. 